Methods in Molecular Biology - Huntington's Disease, chapter 8

\title{
Magnetic Resonance Imaging in Huntington's disease
}

Sarah Gregory, PhD

Rachael I. Scahill, PhD

Geraint Rees, PhD FMedSci

Sarah Tabrizi, FRCP PhD FMedSci

Corresponding Author:

Sarah Gregory

Huntington's Disease Research Centre

UCL Institute of Neurology

$2^{\text {nd }}$ Floor, Russell Square House

10-12 Russell Square

London WC1B 5EH

s.gregory@ucl.ac.uk 


\section{Magnetic Resonance Imaging in Huntington's disease}

Magnetic Resonance Imaging (MRI) is a non-invasive technique used routinely to image the body in both clinical and research settings. Through the manipulation of radio waves and static field gradients, MRI uses the principle of nuclear magnetic resonance to produce images with high spatial resolution, appropriate for the investigation of brain structure and function.

\section{Magnetic Resonance Imaging: A Brief Overview}

\subsection{Measuring the signal}

In MRI, hydrogen typically provides the source of the measured signal due to its abundance within both water and fat. Within the nucleus of each hydrogen atom is a positively-charged proton which spins on its axis, creating a tiny electromagnetic field, known as a magnetic moment (Figure 1a). In an MRI scanner, there is a constant or external magnetic field (known as the field strength of the MRI scanner) in the longitudinal or ' $z$ ' plane which is experienced prior to the administration of a pulse sequence. This causes protons to spin or precess together about the direction of this magnetic field generating longitudinal magnetisation (Figure 1a). To create a detectable signal, however, a second magnetic field is applied that tips protons into the 'transverse' (x-y) plane (Figure 1b). Accordingly, the longitudinal magnetisation is reduced to zero and the protons now precess due to magnetisation in the transverse plane. The ensuing movement induces an electromagnetic current which is measured as the MR signal.

Figure 1 about here

\subsection{Weighting}

As soon as protons are tipped into the transverse plane, longitudinal magnetisation begins to return from zero; this is known as longitudinal or T1 relaxation and characterised by the time constant, T1. Each tissue type has an intrinsic T1 dependent on the surrounding physical and chemical environment. Within cerebrospinal fluid (CSF), for example, molecules move freely, signal intensity is low and consequently their T1 is long; areas containing fluid thus appear dark in the MR image. Conversely, fat tissue has a very short 
T1 and appears bright (Figure 2, left). When protons are tipped into the transverse plane, they interact with each other and begin to lose magnetisation. This is called transverse relaxation and is characterised by a time constant known as T2. Fluids have long T2 relaxation times, due to the freedom of proton movement and are brighter than those tissues, such as fat, which have very short T2 relaxation times (Figure 2, right). For both T1 and T2 relaxation, grey and white matter are clearly distinguishable.

Figure 2 about here

$\mathrm{T} 1$ and $\mathrm{T} 2$ relaxation occur congruently but the relative relaxation time spans can be very different. T1 relaxation can take several seconds, while T2 relaxation can last just a few milliseconds. T1 and T2 weighting fundamentally determines the nature of the image contrast, which represents localised differences in signal intensity due to the physical properties of tissue types. The weighting is determined by the scanning acquisition and in particular governed by the relative combination of two key parameters: TR (repetition time), the time between pulse sequence repetitions, that is, the length of time for which longitudinal relaxation is measured; and TE (echo time), the duration between the initial pulse and the point at which the signal is measured. The degree of T1 and T2 weighting are selected according to the requirements of the scan. For example, T1-weighting is used more commonly for anatomical scans, due to clear boundary definition, while T2-weighting is more commonly used to ascertain pathology. Short TR maximises the differences in T1weighting between tissues. At long TRs, for example, differences between grey and white matter are minimal. TE must also be suitably short to eliminate any differences due to T2 relaxation. T2-weighting is attained by using a long TR to minimise T1-weighting, and a long TE to maximise differences between tissues in the transverse plane.

\subsection{Pulse Sequences}

MRI pulse sequences are a series of radiofrequency pulses and magnetic field gradients that are applied in the scanner and which vary according to the type of scan being collected. An MRI sequence tends to be grouped according to type, with the two most commonly used being Spin Echo (SE), which uses two radiofrequency pulses or Gradient Echo (GE), which uses a radiofrequency pulse followed by a gradient pulse. Both types of sequence produce T1 and T2 weighted images. SE sequences produce high quality images but take longer to 
run compared to GE sequences which are quick to run but can be impacted by magnetic field inhomogeneity (less of an issue in modern scanners). Echo Planar Imaging (EPI) is performed in cases where many scans need to be acquired, such as functional MRI. EPI acquires multiple echoes by rapidly reversing gradients and uses either GE or GE combined with SE. Spatial magnetic field gradients encode the location of all the recorded signals. This signal information is recorded in k-space, a matrix in which raw MRI data are stored and subsequently decoded using the Fourier transformation to produce an MR image.

\subsection{Scanner Hardware}

MRI scanners have different magnetic field strengths. Most commonly the 1.5 Tesla (T) is used clinically and the 3T for research. Field strength determines the overall size of the signals recorded and therefore ultimately the image quality, with higher field strength resulting in better image quality. $3 \mathrm{~T}$ is preferable for research because of the quality of the images necessary to detect subtle changes, which can be collected in a considerably shorter time. However, 3T images are more likely to be impacted by artefacts due to motion, susceptibility (particularly in frontal or temporal lobes), blood or fluid movement (see later section).

\section{MRI modalities used in HD research}

\subsection{Structural MRI}

Structural MRI provides information regarding the anatomy and pathology of the brain. It is generally used for the quantitative measurement of morphological information including volume, cortical thickness and surface area. To ensure that specific structures and boundaries can be identified correctly it is necessary for structural images to have good tissue class contrast, high spatial resolution and as little motion artefact as possible. Most structural images are T1-weighted as this gives the best between-tissue contrast. Additionally, an optimal balance between high spatial resolution and signal to noise ratio needs to be achieved. This is determined when selecting acquisition parameters, including voxel size $\left(\mathrm{eg} 1 \mathrm{~mm}^{3}\right)$ to improve resolution, slice thickness and scan length. 
Structural MRI has been used widely in HD research to investigate volumetric change. This can be achieved using whole brain techniques, such as Voxel Based Morphometry [1,2] (see section below) or manual segmentation of specific structures [3]. These methods have been used to investigate changes in cortical and subcortical volume both between groups and longitudinally and have shown a highly robust and consistent pattern of reduction in striatal (caudate and putamen) volume as HD progresses [4-11] (Figure 3). Other studies have investigated changes in cortical thickness $[12,8,13]$. Cortical thickness measures the thickness of the cortical mantle, between the pial surface and the white matter, and allows the comparison of cortical thickness while accounting for the nature of the cortical surface; cortical folding, for example, cannot be accounted for in standard volumetric analyses.

Figure 3 about here

\subsection{Diffusion Weighted Imaging}

Diffusion Weighted Imaging (DWI) investigates white matter microstructure within brain tissue by examining the effect of tissue organisation on water movement [14-16]. Measurements of axonal orientation and ease of diffusion can be used to infer the underlying nature of fibre integrity.

Water movement in free space is isotropic, it can move in any number of directions. However, along the main axis of a white matter fibre diffusion occurs in one direction and diffusivity is inherently anisotropic. The diffusive properties of water contribute to the attenuation of the MR signal, such that the greater the freedom of water movement within a voxel, the greater the reduction in signal. For example, in dark areas containing CSF, water is isotropic, protons are displaced more rapidly and accordingly the MR signal decreases rapidly. Conversely, areas where voxels are brighter indicate restriction of water movement and so greater MR signal; this is clearest in white matter.

Diffusion Tensor Imaging (DTI) was introduced as a more efficient and accurate means of measuring anisotropy due to its independence of orientation within the scanner [17]. The diffusion tensor measures diffusivity along the three principal fibre directions (or axes) within a voxel. The additional measurement of 'off-diagonal' elements enables measurement that is independent of the frame of reference. The diffusion tensor is characterised by three 
eigenvalues $\left(\lambda_{1}, \lambda_{2}, \lambda_{3}\right)$ which represent diffusivity along the three principal axes and three eigenvectors $\left(\varepsilon_{1}, \varepsilon_{2}, \varepsilon_{3}\right)$ which represent corresponding orientation (Figure 4). The principal eigenvector, $\varepsilon_{1}$ is assumed to run parallel with the dominant underlying fibre direction. Once the tensor has been estimated a number of indices can be derived, namely the trace and anisotropy measurements. The Fractional Anisotropy (FA) index captures the degree of anisotropy within a white matter tract and FA maps are derived from the estimated tensors where the brighter the voxel intensity, the greater the degree of FA. Mean diffusivity (an average of diffusivity in all three directions) (MD), parallel (axial) diffusivity (AD) and perpendicular (radial) diffusivity (RD) are also often interrogated.

Figure 4 about here

DTI data can be analysed using either a whole brain or regional/tract-based hypothesisdriven approach. Techniques such as Tract-based Spatial Statistics (TBSS) [18] are used to investigate changes in white matter microstructure across the whole brain, while tractography examines diffusivity within specific tracts [19]. More recently, studies have utilised whole-brain tractography approaches to investigate structural networks across the brain [20-22].

DTI has been used widely in HD research. After initial mixed findings, it has been shown that in HD, FA is generally lower in widespread areas, with radial or perpendicular diffusivity also increasing from the premanifest stage and AD beginning to increase as the disease progresses [23-36]. This evidence points to increasing disorganisation of white matter microstructure in HD.

\subsection{Functional MRI}

Functional MRI (fMRI) is used to investigate brain activity during a task or spontaneous activity occurring at rest. During task-fMRI participants perform a task or function while their brain activity is recorded. In contrast, resting-state fMRI, investigates spontaneous activity when the brain is at rest.

fMRI measures signals related to the neurovascular coupling of neural activity and blood flow. BOLD (Blood Oxygen Level-Dependent) fMRI measures a signal related primarily to blood oxygenation, which in turn reflects metabolic activity in the tissue [37,38]. Discrete 
increases in brain activity occur as blood flow to a region of activity is increased. When haemoglobin becomes deoxygenated, it becomes paramagnetic relative to oxygenated haemoglobin, and so changes in the proportion of oxy- to deoxy-haemoglobin due to local neuronal activity produce measurable changes in the BOLD signal. The vascular origin of the change in signal has a time course (known as the haemodynamic response function, HRF) which is slow compared with that of underlying neuronal activity [39], but the 'neurovascular coupling' of blood flow and increased neuronal activity can provide accurate localisation of neuronal activity [40]. Signal changes within or about smaller vessels contribute to the high spatial specificity associated with fMRI. As with structural imaging, a trade-off between increasing spatial resolution and signal to noise ratio needs to be determined prior to the beginning of a study.

fMRI data are analysed using a number of approaches. For task-based analyses, the fMRI signal is convolved with the HRF and the putative underlying neuronal activity matched to different components of a task. Contrasts between task conditions are created to pinpoint the location of task-associated brain activity and then analysed at the group level. Resting state fMRI data can be analysed in a similar way, but as there is no task involved, restingstate analyses focus on connectivity between regions in the brain at rest. Functional connectivity investigates temporal correlations in activity across every voxel in the brain. Seed-based connectivity analyses identify temporal correlations between activity in a prespecified region and the rest of the brain while Independent Component Analyses uses a data driven approach, identifying groups of regions or networks clustered together according to temporal correlations in their activity. Functional connectivity does not allow for inferences about causality and underlying mechanisms, however, as it does not provide directionality of effect. Effective connectivity can be used to test the causal effect of activity in one region on the activity in another. This can be implemented using techniques such as Structural Equation modelling [41], Granger Causality [42] and Dynamic Causal Modelling [43].

Task fMRI has been widely used in HD research to explore the changes in task networks particularly associated with the HD phenotype, primarily motor and cognitive. Studies have shown areas of hypo- and hyper- activity associated with both task networks in HD groups 
when compared to controls or HD groups of differing disease stage [44-51]. It has also been shown that increased activity in some areas may reflect compensatory activity $[52,50,53]$. Resting state fMRI studies have also explored networks in HD gene-carriers while at rest; these have included, in particular, the default mode network (task-negative activity in the brain at rest) and attention networks $[54,47,55,56]$. Although not consistent, evidence suggests that there are HD-specific changes in the brain networks at rest.

\subsection{Considerations when undertaking an MRI study}

Undertaking an MRI study can be challenging. Each imaging technique has specific requirements that we will discuss throughout the chapter, but there are a number of factors which must be considered for any MRI study in a clinical population.

A good study design is vital for achieving good MRI data. For structural imaging, it is important to ensure that scans have full coverage of the brain and to minimise the chance of artefacts due to motion and local magnetic fields (see below). For task fMRI sequences, the task must be specific to the hypothesis being tested as data cannot generally be used to answer alternative questions. It is also important to ensure that the task is feasible for the scanner environment, that is, sufficient information can be captured in a short period of time. It is important to work closely with the physicist and / or radiographer to ensure that the MRI sequence will optimise data collection allowing for the best data possible.

For all types of MRI data, longer scan time increases the number of scan volumes collected which, in turn, tends to increase statistical power. The number of participants in a study is typically more important than the number of scans collected per participant as interparticipant variability tends to exceed inter-scan variability. Power calculations are more challenging in the context of MRI studies as the size of any effect can vary considerably according to modality and nature of the experiment [57,58]. It is useful to look at previous studies for guidance, but it is important to remember that even subtle differences in processing pipelines used to analyse MRI data can have a considerable effect on outcomes. There are a number of steps that can be followed to ensure congruency between experiments [59]. 
When designing the protocol for a scanning session, it is important to choose the right order of scans. It is sensible to put the most important scans at the beginning, should a session overrun or have to be terminated early. Researchers must also consider the stress of a long scanning session for a participant and it is important to factor in breaks if necessary. However, to avoid possible session effects and disturbance of the scanner equilibrium (alignment of magnetisation with static magnetic field), it is advisable to keep these to a minimum.

Within the scanner, it is important that participants remain as still as possible. Movement artefacts can cause MRI images to be distorted and ultimately discarded. Head movement of only half the width of a voxel can lead to a mixture of signals from two different anatomical or functional brain regions. This is naturally challenging when scanning patients with a movement disorder, particularly if advanced. The use of head cushions can help, but it is also the responsibility of both the researcher and radiographer to convey the importance of remaining still and to try to combat any potential anxiety. This can be done by explaining the nature of the session beforehand, keeping in constant contact with the participant and ensuring that the patient is comfortable by using padding. It is also important to establish if a participant has claustrophobia prior to the scanning session. Minor head movement is generally unavoidable, however, so rigorous quality control of the raw data is essential.

\section{Data Acquisition}

Given the strength of the magnetic field, safety is always paramount in the scanner environment. All metal must be removed prior to any participant or researcher entering the scanner room and safety checks are required to verify absence of metal in the body, tattoos or cosmetic make up and whether there is a possibility of pregnancy (which is usually a contraindication to MRI scanning).

Structural MRI sequences are devised to maximise contrast between different tissues with T1-weighted images are most appropriate for this purpose. The sequence and acquisition parameters will depend on the structures of interest. The image contrast in DWI reflects the differences in rate of diffusion between tissues. Diffusion imaging utilises a basic spin-echo sequence adding a diffusion-encoding gradient either side of the spin echo refocusing pulse. 
All DWI images have T1 and T2 weighted contrast in addition to the intended diffusion contrast. fMRI imaging requires the rapid acquisition of a large number of images to ensure accurate measurement of physiological changes. This is most commonly achieved using EPI [60]. The BOLD effect is measured using differences in T2* decay; this is decay due to proton-proton interaction plus field inhomogeneity and can be manipulated by changes in TE. As spin echo effectively eliminates $\mathrm{T}^{*}$, gradient echo sequences are more appropriate for fMRI scanning. During an fMRI imaging session, a series of images are collected across a time course. For task fMRI, a time-series comprises a number of runs composed of taskrelated epochs. These are time-locked periods during which an event of interest occurs.

\section{Data Quality Control}

There are a number of quality control steps that should be carried out prior to data preprocessing for all MRI modalities. It is advisable to perform quality control after each scanning session, so that any acquisition errors can be corrected for future scans.

Data are firstly converted from DICOM format used by scanners into either ANALYZE (.hdr and .img) or more likely into NIFTI (.nii) format for visualisation. Initial investigation for gross artefacts checks motion, brain coverage, large artefacts or areas of signal dropout. In MRI there are a considerable number of potential artefacts; the main being motion, susceptibility, flow and inhomogeneity (Figure 5). Motion artefacts are probably the most common and impact all types of scanning. General movement can cause gross artefacts where it looks like the image has been printed multiple times (Figure 5a). Physiological motion, nominally cardiac and respiratory can cause ghosting on images, which worsens with movement and the signal intensity from the particular tissue. Flow (blood flow) and the presence of new protons can enhance or diminish a signal also leading to ghosting. Wrap around artefacts occur when anatomy appears outside the field of view and appears as if part of the anatomy has been folded over or displaced. Susceptibility and metal artefacts are also highly common and are related to large change in magnetic susceptibility (Figure 5b). Artefacts due to metal are very evident causing a dark region and highly intense regions where the metal interfaces with the body tissue. This can also cause distortion in the neighbouring tissue and can be largely prevented prior to scanning. Susceptibility artefacts 
arise due to different tissues having different magnetic susceptibilities that can cause the signal to drop very quickly and create black regions on an image. There are also a number of hardware-related artefacts, such as magnetic field inhomogeneity which cause distortion. Magnetic field inhomogeneity artefacts are caused by a magnetic field that is not completely uniform. This inhomogeneity results in varying image contrast which can impact processing of the data. However, the artefact can be corrected via a process called bias correction. This correction results in more uniform intensity across the MRI scan, and the most frequently used algorithm is the N3 method [61].

Figure 5 about here

\subsection{Modality Specific}

All of the above artefacts can affect MR images regardless of the scanning sequence. There are, however, some checks that are more specific to the type of data collected. For structural images it is important to check the quality of the tissue contrast. For DTI, there are no consensus guidelines for removing particular volumes due to movement or artefact, but removing a dataset in its entirety rather than a handful of volumes may be preferable as each volume measures signal in a different direction; removing corrupted volumes specific to each participant could lead to uneven sampling. For fMRI, it is important to exclude participant data where there are large areas of signal dropout as this ultimately restricts the search area for statistical testing. It is possible to check between volume and between slice variability and the signal to noise ratio for each scan, for example (http://imaging.mrccbu.cam.ac.uk/imaging/DataDiagnostics). There are a number of automated programs now that present a whole series of information regarding slice and voxel signal to noise ratio (fBIRN) (https://www.nitrc.org/projects/fbirn/).

\section{Pre-Processing Data}

MRI data are pre-processed and analysed using a number of different packages with the most common being Statistical Parametric Mapping (SPM) (http://www.fil.ion.ucl.ac.uk/spm/) which works on a Matlab foundation (uk.mathworks.com/products/matlab), and FMRIB Software Library (FSL) (http://fsl.fmrib.ox.ac.uk/fsl/) based on a Linux platform. Throughout the chapter, we will 
refer to these two main software packages as they are multifunctional and routinely utilised. There are also other software packages such as Freesurfer (https://surfer.nmr.mgh.harvard.edu/) which are used for a specific modality analysis.

There are a number of pre-processing steps that are applied to most types of MR data regardless of modality and we will discuss these in more detail here.

\subsection{Segmentation}

During this step, image data are partitioned and classified into three main tissue types (clusters): grey matter, white matter and CSF. The process of segmentation divides an image into a set of homogeneous (intensity, depth, colour, or texture) regions which are then assigned a tissue class (defined in advance). The "labels" are typically limited to WM, GM, CSF, brain and air, in addition to some mixtures of classes. Regions are usually coherent continuous shapes, such that neighbouring voxels are likely derived from the same tissue class. It should be noted that there is a degree of circularity within this process given that segmentation motivates tissue classification while the classifier is used to segment an image. A Bayesian classifier in conjunction with the Expectation Maximisation algorithm has been adopted in most packages including SPM and FSL. In SPM, for example, segmentation uses a modified mixture model cluster analysis technique, whereby tissues are classified according to both the intensity and spatial distribution of voxels. Manual segmentation of tissues or regions is also commonly used, particularly for deep structures such as the caudate, which are more challenging for automated algorithms. However, this is highly labour intensive.

\subsection{Normalisation}

The process of normalisation accounts for differences in gross anatomy and brain size across participants by transforming all images into the same reference space. Reducing macroscopic differences ultimately creates a focus on local tissue composition and smaller structures. Using deformation fields, individual brains are mapped to a standardised template using a least-squares fit model. This measures the extent to which individual brains deviate from that of the template by minimising the difference between the sum of squared residuals (error values) from the input images and the template. Normalisation encompasses both macroscopic (linear) and more localised individual (non-linear) differences. The typical 
SPM approach is to register global structures of individual images to a template using an optimum 12 parameter affine transformation, during which images undergo translation, zoom, shear and rotations in each plane $(\mathrm{x}, \mathrm{y}, \mathrm{z})$; optimum parameters are then estimated using least-squares minimisation. Non-linear registration accounts for gross individual differences between images. Exact matching between scans, however, is not desirable, as this would effectively eliminate any distinction between the two groups. There are also alternative surface-based normalisation techniques used by packages such as FreeSurfer.

\subsection{Smoothing}

This is the final step in the processing of data prior to analysis. This process improves signal to noise ratio and in averaging the number of observations across groups of voxels the signal becomes increasingly normally distributed, which is necessary for parametric statistical testing. The smoothing process is carried out using an isotropic Gaussian kernel, so-called because it implements a Gaussian or normal distribution across voxels such that signal is average-weighted for each voxel. The size of the kernel dictates the area over which signal averaging occurs and characterised by Full Width Half Maximum (FWHM). The kernel is applied to each data point. It is important to note that there is a trade-off between increased smoothing to make the data more normally distributed and reduce noise and a decreased ability to spatially localise the signal. The optimum dimensions of the kernel are, therefore, selected according to the resolution of the data and the size of hypothesised regional differences.

\subsection{Modality Specific Structural MRI}

Here, we will focus on two of the most commonly used techniques: volumetric analyses using Voxel Based Morphometry (VBM) and cortical thickness analyses using Freesurfer software. Pre-processing pipelines will vary according to the analyses planned.

\subsubsection{Voxel-Based Morphometry}

Processing for VBM requires three key steps: normalisation, segmentation and bias correction [62]. Unified Segmentation combines normalisation, segmentation and bias correction into one circular, integrative model. Intensity within each cluster is modelled using Gaussian distributions that are characterised by three parameters: the mean signal 
intensity across voxels within a cluster, the variance around the mean signal intensity and the number of voxels within the cluster. These parameters are estimated for each image using an iterative 6-step algorithm, which involves repeatedly estimating and updating the belonging probabilities of voxels to a particular tissue type. Prior probability maps which outline spatial distributions and the likelihood of a voxel belonging to a specific tissue class regardless of signal intensity are included, while the use of Hidden Markov Random Fields allows the incorporation of information regarding the tissue class of neighbouring voxels. The parameter estimation uses an Iterated Conditional Modes (ICM) approach, which optimises individual parameters iteratively, while maintaining others at their best fit.

VBM examines differences in grey or white matter based on either density or concentration measurements of tissue volume. In warping the individual images to standardised space, cortical structures are expanded and contracted to match that of the template image. This has the effect of adjusting individual volume measurements and only relative regional values can be examined. This can be useful, but it does not permit an absolute comparison between groups. An additional step can be introduced into the processing, which allows for absolute volume comparisons. The warping parameters used to map the individual images onto the template are multiplied by the new warped grey matter volumes to ascertain absolute differences in volume. Both modulated (volume) and unmodulated (concentration) can be analysed. Images are then smoothed as described earlier.

\subsubsection{Cortical Thickness}

FreeSurfer uses a surface-based pipeline to measure and analyse features such as cortical thickness, surface area and curvature of the brain (a volume-based stream calculates volume and classifies subcortical regions [63-65].

Structural images are initially bias corrected for field inhomogeneity, registered using affine transformation to the MNI305 atlas and finally skull stripped. White matter voxels are then identified based on their intensity, position and surrounding voxel intensity and a triangular mesh is constructed around the white matter, which is smoothed and topologically corrected. The external cortical surface is created by expanding the white matter mesh until a point of maximal contrast between grey matter and CSF is reached. 
Volume can also be measured with FreeSurfer by using a separate volumetric processing stream. The brain is affine registered to MNI305 space, and an initial volumetric labelling step is performed followed by bias correction. It is then registered using a high dimensional nonlinear volumetric alignment to a Talairach atlas. The image is then labelled.

\subsection{Diffusion Tensor Imaging}

DTI data includes a series of diffusion weighted images each acquired using a different gradient direction and one image which has no DTI weighting - the B0 image, in addition to files containing gradient direction information to be used in the processing.

There are several necessary pre-processing steps. First, the data should be corrected for the eddy currents that are induced due to changing electromagnetic fields and worsened with motion [66]. These currents can cause distortion in the images and therefore, it is standard practice to correct for them; optionally updating gradient direction information accordingly. The tensor is then estimated; with the shape and measures of tensor determined by the different gradient directions. The tensor is estimated for each voxel using multivariate linear regression of signal intensities. It is important to check the outputted diffusion tensor maps to ensure that the fibres are delineated appropriately in each of the three planes.

Diffusivity metrics can then be extracted.

For tractography analyses, it is necessary to perform additional pre-processing steps. The B0 image may be registered to the T1 image in cases where tractography is performed in an individual's structural space. Most importantly, crossing fibres should be managed [67]. Each voxel contains a large number of fibres oriented in different directions and it is important to exclude fibres of no interest from the final tracking.

\subsection{Functional MRI}

For both task-based and resting state fMRI, data are processed in a similar way.

\subsubsection{Realignment}

Due to individual head motion in the scanner, head location will vary across volumes within a time series. Using rigid body transformation, realignment places all images in alignment with the first to ensure that composition of each voxel is consistent across the time series. As 
the shape and size of the brain does not change between images of an individual, six parameters (three translations and rotations) are adequate for intra-participant registration. Adjustment is ascertained using an iterative algorithm based on best-fit cost function.

\subsubsection{Co-registration}

The functional images have poor contrast and identification of regions of interest can be difficult. Consequently, the time series is co-registered to a high-resolution structural image that has been collected during the scanning session from the same participant. As in the realignment process, a six-parameter rigid body transformation can be used to achieve coregistration of a time series to the structural image using a cost function based on mutual information. However, because of possible geometric distortions that can occur within EPI pulse sequences, an additional three parameters are included which account for scaling along the axes. The time series can then be resliced to match the first image on a voxel-byvoxel basis.

\subsubsection{Normalisation}

The structural image is first warped to a template image. The deformation parameters derived from this normalisation process are then used to warp the entire time series of fMRI images into standard space which enables statistically sound comparison across participants.

\subsubsection{Smoothing}

The Gaussian smoothing kernel is chosen according to the size of the expected signal of interest (this maximises signal to noise ratio). In the case of fMRI, spatial correlations with neighbouring voxels exist based on functional similarities. If the filter is matched to the size of expected correlations, this improves the ratio of signal to noise within a voxel without impinging on spatial resolution quality.

\section{Data Analysis}

\subsection{General Concepts}

\subsubsection{Statistical Analysis}


Statistical analysis of imaging data is generally underpinned by the General Linear Model (GLM). The GLM uses multiple regression to investigate the contributory effect of one or more regressors or independent variables to the outcome of a dependent variable using the basic formula $Y=X \beta+e$; where $Y$ represents the dependent variable; $X$ the regressors or independent variables; $\beta$ the estimated parameter values of these regressors (weighted contribution to the output) and e the error or residual values (the extent to which each individual dataset varies from the mean). Within the context of MRI, activation or tissue type are estimated at each voxel using a variant of the GLM, whereby the standard GLM equation is replaced by a set of matrices. The design matrix includes the regressors for each time point and is multiplied by the parameter matrix, which weights the regressors in terms of their contribution to the retrieved signal (parameter weights). Model estimation identifies to what degree the hypothesised time course actually contributed to the real data. If parameter weights are high the contribution of the regressor to the BOLD signal is substantial. Also included in the design matrix are co-variates of no-interest or nuisance regressors, such as age or IQ; the contribution of these variables is eliminated during the final stage of analysis.

High and low pass filters are utilised to remove any unwanted signal frequencies, which indicate noise interference and potential signal distortion. By introducing a filter, frequencies which correspond to noise, for example, respiratory, can be eliminated while maintaining those associated with the signal of interest. Low pass filters attenuate high frequencies, and the high pass filters low frequencies.

\subsubsection{Contrast Weighting}

Contrast describes the extent to which experimental manipulation contributes to a change in activation or volume, for example, providing a linear combination of estimated parameter weights for a group or condition. In a simple two-condition fMRI study, for example, if we wanted to investigate the extent to which condition one impacted on the BOLD signal, a simple $(1,0)$ contrast would be tested. This multiplies the estimated parameter weights for condition 1 by the contrast vector, while scaling the value according to the extent of the error function. The zero weighting for the other condition means that the resulting statistic does not account for any specific contribution of condition 2. Comparisons between conditions or 
groups provide a more robust indicator of the independent variable manipulation. Using the above example, to compare condition 1 with condition 2 , the contrast vector would be $(1,-1)$. This is equivalent to areas of the brain where activations are higher in condition 1 compared to condition 2. Contrast represents the statistical evaluation of whether an experimental manipulation evokes a significant change in activation or volume.

At the first level, within-participant analysis is performed, for example, a task contrast may be run for a particular comparison for each individual. At the second analysis level, these individual contrasts will be inserted in a design matrix to examine group differences or correlation and similar contrasts used to represent groups rather than conditions. Provided normality assumptions are satisfied, least square estimation is used to ascertain maximum likelihood estimates for residuals, creating a model of best fit. A t-statistic is calculated for the contrast or parameter difference within each voxel based on the contribution of parameter weights and the scaling of residual error evaluated against a null hypothesis of zero. The combination of all the t-tests for every voxel is presented as a statistical parametric map (SPM), which details those voxels significant for the contrast weighted comparison.

\subsubsection{Multiple Comparisons Correction}

Typical brain images comprise between 50,000 and 500,000 voxels (approximately) depending on the spatial resolution. The large number of voxels greatly increases the number of statistical comparisons and therefore the possibility of type 1 errors. Therefore, we must correct for multiple comparisons. Bonferroni corrections are inappropriate for imaging, as they assume independence for every statistical test; this is not the case given the spatial correlation (tissue belonging or activation), which exists between neighbouring voxels. The False Discovery Rate (FDR) is the preferred less stringent method for multiple comparisons correction as it correlates the probability of a false positive in relation to the number of positive results, using the alpha value. However, implicit in the application of the Gaussian (normally distributed) fields is a multiple comparison correction conducted which accounts for this non-independence [68,69]. Gaussian random field (GRF) theory is used to estimate the number of independent statistical tests or resolution elements (resels) within a statistical parametric map. Based on the number of resels, the number of activation clusters derived by chance at a specified statistical threshold can be determined; this is known as the 
expected Euler characteristic. Using GRF theory, the analysis package determines the statistical threshold whose expected Euler characteristic corresponds to the alpha value; this provides corrected statistical values. Input threshold at an uncorrected level is commonly $\mathrm{p}=0.001$, with corrected values of $\mathrm{p}=0.05$.

\subsubsection{Inference}

For a group of participants, there is a fundamental distinction between making inferences about an effect in relation to within-participant variability or with respect to betweenparticipant variability. This distinction relates directly to the difference between fixed and random-effects analyses. In a fixed-effects analysis, the error variance is estimated on a scanto-scan basis, assuming that each scan represents an independent observation. The degrees of freedom are essentially the number of scans. In a random-effects analysis, the appropriate error variance is based on the activation from participant to participant where the effect per se constitutes an independent observation and the degrees of freedom falls substantially to the number of participants. The term 'random-effects' indicates that the researcher has accommodated the variability of differential responses by comparing the mean activation to the variability in activations from participant to participant. Both analyses are valid, but constrain the type of inference. Thus, inferences based on fixed-effects analyses are about the particular participant studied. Random-effects analyses are typically more conservative but allow the inference to be generalised to the population from which the participants were selected.

\subsection{Structural Imaging}

\subsubsection{Voxel based Morphometry}

VBM analysis uses Statistical Parametric Mapping to investigate volume. Within standard VBM processing, cluster wise analysis is inappropriate due to the variability of smoothness across the brain; large clusters are apparent in very smooth areas, leading to false positives, while small clusters in less smooth areas could result in Type 2 errors. However, there are software toolboxes which have adapted the process and allow for cluster inference (http://www.neuro.uni-jena.de/vbm/). Output images describe regions in which volume is greater in one group compared to another or correlated with a co-variate of interest. Within 
SPM, images are initially statistically thresholded then cluster inference (if used) will show those clusters, voxels and peaks that are significant at an uncorrected and corrected level (Figure 6a).

Figure 6 about here

\subsubsection{Cortical Thickness}

FreeSurfer uses the GLM to examine surface-based measures such as cortical thickness or gyrification. The GLM is used to test how measures may vary with varying demographic or group variables. Variables can be included in the model as extraneous factors. Multiple comparisons are typically controlled for via FDR correction, however a more complex method using simulated data to perform Monte Carlo correction for multiple comparisons is also available. The data can be shown in plots and images that display significant associations mapped onto an average subject. Values for cortical thickness, gyrification and other measures can also be extracted for different regions in a text file and analysed using other statistical methods and software independently to FreeSurfer.

\subsection{Diffusion Tensor Imaging}

\subsubsection{Tract Based Spatial Statistics (TBSS)}

TBSS is performed using FSL software and comprises a four step process [18]. The TBSS analysis works on the FA images that were created following tensor fitting. All FA images from all participants are aligned in a standard space using either a customised template, which is warped into standard space, or more commonly each individual image is warped to an FA standard-space image within FSL. All the FA images are then merged into a single $4 \mathrm{D}$ image file and a mean image created. This mean image is then used to create the mean FA skeleton on which the statistical analyses are performed. In a good example, the main tracts should align well with the FA skeleton for each participant. Statistical analysis is then performed using a specified threshold to include voxels with mean FA of (usually) 0.2. This creates a binary skeleton mask that defines which voxels will be included in the statistical analyses and each participant's FA data is projected onto the skeleton creating a 4D image. The images are then ready for group analyses. FSL uses a GLM tool which performs nonparametric permutation tests to investigate which voxels within the skeleton differ 
significantly between the groups. FSL uses a clustering tool, comparable to that used in SPM, called Threshold-Free Cluster Enhancement (TFCE) [70]. When fitting the tensor, not only are FA maps outputted, but also Mean Diffusivity, Axial and Radial Diffusivity maps. TBSS can be adapted to test for differences in all of these metrics. Group differences are visualised by overlaying the statistical maps onto the mean FA skeleton overlaid onto a structural image (Figure 6d).

\subsubsection{Tractography}

Tractography tends to be performed using one of two types of algorithm: deterministic or probabilistic [71]. Deterministic fibre tracking was used in early tractography studies and works on the principal that the main eigenvector within each voxel represents the existent underlying fibre orientation in a continuous fashion. Pathways are tracked from an initial seed point or region of interest (ROI) that intersects this tract and reconstruction occurs in a step-wise fashion moving in the direction of the principal eigenvector at a sub-voxel level, commonly $0.5 \mathrm{~mm}$. At each step the tensor is estimated, diagonalised and the next step taken in the direction of the highest diffusivity. Drawbacks attributed to deterministic tractography are that only one trajectory can be constructed per seedpoint. More importantly, there is always a degree of uncertainty associated with the estimation of the tensor, and its representation of the underlying fibre orientation. This is particularly significant in areas where there is not one dominant pathway. In contrast, probabilistic algorithms propagate a series of pathways from one point of origin. The algorithm assumes that there is no continuous field, so that while fibre orientation within a voxel is uniform, at the voxel boundary there are a number of possible directions in which the tract could be manoeuvred resulting in multiple pathways reconstructed from the same ROI. Percentage values are then assigned to the streamlines passing through each voxel. For both methods, thresholds based on a fixed anisotropy value are imposed to prevent the tracts from entering into grey matter. Angular restriction prevents unlikely fibre orientation and provides termination criteria. Tractography is heavily dependent on data quality and the efficiency of the modelling process (Figure 6b).

The most important element of tractography is deciding on the tracts of interest, usually based on clinical or empirical evidence. In the case of $\mathrm{HD}$, it is practical to look at motor or 
cognitive pathways. It is most common to define seed ROIs using standardised atlases or masks, which are then warped into individual native space. It is important to ensure that placement of all regions for each individual is anatomically sensible and to have some knowledge of the anatomy of the tract itself. Exclusion masks both in regions of the brain and in the grey matter are necessary to ensure that only fibres within the tract of interest are reconstructed and that the tracking does not extend beyond white matter into grey matter or dura. Diffusion metrics are extracted from the reconstructed tracts and included in a standard statistical group analysis.

\subsection{Functional MRI}

\subsubsection{Task-based analyses}

The basic principle of experimental design in fMRI is to manipulate the participant's experience or behaviour in a way likely to produce a functionally-specific neurovascular response. It is important to devise an experimental paradigm which is both suitable for implementation within the scanner and which effectively tests the hypothesis. Two types of experimental design are in common use: blocked design and event-related design. Blocked design consists of long interval blocks (on average 20-30 seconds in length) of 'on' periods during which stimuli for an experimental condition are presented and 'off' periods of rest (baseline). This format allows simple between-condition and condition-baseline comparisons. Activations of no interest that occur in response to other aspects of the task are matched in each condition and thus eliminated. Blocked designs do not measure responses to individual stimuli; by using long task intervals steady state activation is achieved which represents ongoing neuronal activity throughout each block. This leads to a robust haemodynamic response function (HDR), which models delivery of blood to tissue following neuronal stimulation. However, with blocked design cognitive function during a rest or baseline block cannot be controlled, while responses relating to individual stimuli cannot be determined.

Event-related (ER) designs are focused on changes across time and measure activity of interest in short and discrete intervals. Individual trials are presented in a random order (jittering) separated by an inter-stimulus interval; epochs, which are time locked to an event of interest, are extracted and then averaged according to similarity of events (at the first - 
level). According to linear functionality, each stimulus interval elicits an independent HDR representative of the neural activity related to the stimulus. ER designs are much more flexible than the blocked designs, because they allow for randomised presentation of timings of the specified stimuli. The main disadvantage of event-related design is that signal changes induced by a single trial are generally weaker than signal changes typical in a blocked experiment. It is, therefore, often necessary to increase the number of experimental trials to improve statistical power, invariably leading to longer scanning time.

Data are analysed using the GLM at both the first (individual) and second (group) level. The output images describe those regions in which activity is greater in one group compared to another (according to the contrast weighting specification) or correlated with a co-variate of interest. As with VBM, images are statistically thresholded for cluster inference, both at an uncorrected and corrected level (Figure 6c).

\subsection{Resting-state analyses}

For resting state data analyses, the main focus is on connectivity. It is possible to perform functional connectivity analyses on task data, but it is more commonly used to examine temporal correlations between activity in regions across the brain at rest. Effective connectivity, which looks at underlying causal mechanisms and the effects of one region on the activity of another can also be examined.

\subsubsection{Functional Connectivity}

There are two main ways that functional connectivity is examined in resting state data: hypothesis-driven seed connectivity and data-driven independent components analysis.

Seed-based connectivity is a simple analysis, whereby an ROI is selected, in the case of HD possibly a motor region, which is defined by using a set of co-ordinates from the literature or a mask $[72,73]$. The average time series is then extracted from this region and a brainwide, voxel-wise analysis run to identify in which voxels the temporal pattern is the same as that of the region of interest, i.e. temporally correlated. This would reveal resting state networks that involve the seed region and which emulate functional networks seen when the brain is active in a task or function. Individual maps can then be used for group level analyses. Resting state fMRI data is generally more noisy than task data because it is low 
frequency incorporating physiological (non-neuronal) signals, and there are no contrasts (as per task fMRI) by which non-neuronal noise is largely edited out. Therefore, it is advisable to remove as much non-neuronal noise as possible. The simplest and crudest method is to include sample time-series from white matter and CSF within the first level analysis, thus modelling (and ultimately removing) the majority of cardiac and respiratory noise.

Preferable is to record and model respiratory and cardiac measures whilst the patient is in the scanner. Finally, it is recommended, although a matter of some controversy, not to regress for global signal as is standard [74-76]. This has been shown to cause a predisposition towards negative correlation in analyses.

Independent Components Analyses (ICA) can be run using the GIFT toolbox associated with SPM [77] , MELODIC in FSL [78], or a number of other toolboxes and software packages that are available. ICA is data driven and is a blind source signal separation technique in which a series of mixed signals each relating to a particular activity or noise within the fMRI data are decomposed into time series that are correlated over time with corresponding spatial maps. In the case of resting state fMRI these maps depict functional networks or noise; the latter of which can be ignored or modelled as unwanted variance. ICA can be performed for an individual to classify and remove structured noise components from data. Alternatively, ICA is conducted on concatenated sets of many individuals' data from a particular group and each ensuing component represents variance across each individual, time and space. Dual regression analysis allows component maps to be regressed back onto individual patient data for group analyses $[79,43]$.

\subsubsection{Effective Connectivity}

Effective Connectivity investigates directional effects between regions where functional connectivity simply investigates non-directional relationships. Understanding direct causality is important in the development of therapeutics; knowing which regions cause degeneration in others. There are a series of effective connectivity measures which can be applied including Structural Equation Modelling [41] and Granger Causality [42], but a relatively recent technique called Dynamic Causal Modelling (DCM) [43] is used to model empirically-driven networks in order to infer the causal architecture of distributed dynamical systems. DCM can be used with both task and resting space fMRI and the key 
factor is selecting the most relevant regions for a biologically-plausible network. Different models of potential causality (eg. top down or bottom up processing) are then modelled using time series data extracted from each region and estimated using a model of underlying neuronal activity. This process then identifies which model best explains the data. Connectivity values can then be extracted from each individual and compared across group or included in a correlation analysis.

\section{Acknowledgements}

The authors would like to thank Eileanoir Johnson for her suggestions and comments. 
Figure Legends
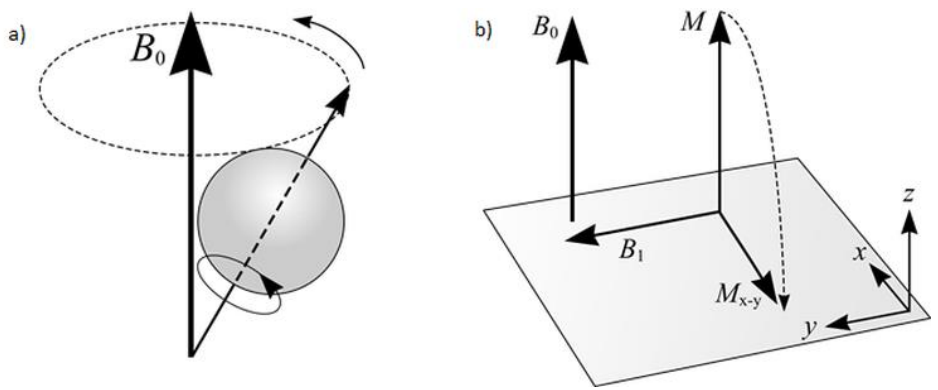

Figure 1: Magnetisation. a) Magnetic Moment: the path of a hydrogen proton precessing about a central axis; b) Application of gradient pulse: protons with magnetisation in the $\mathrm{z}$ plane $(\mathrm{M})$ generated by the presence of an external field (B0) are tipped into the $x-y$ plane following application of second magnetic field (gradient pulse) creating transverse magnetisation $\left(\mathrm{M}_{x-y}\right)$. Reproduced from voxelcube.com with permission from Mike Puddephat, 2016.

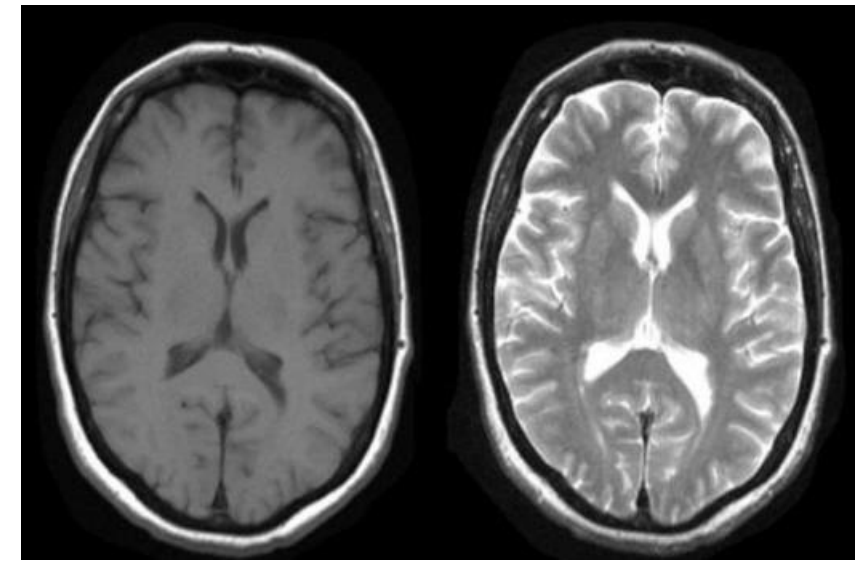

Figure 2: T1 and T2 weighting. Left: T1 weighted image - areas containing fluid appear dark, while fat tissue appears bright. Right: T2 weighted image - areas containing fluid appear bright, while fat tissue appears darker. Reproduced from svuhradiology.ie/diagnostic-imaging, with permission from Eric Heffernan, 2016. 
.
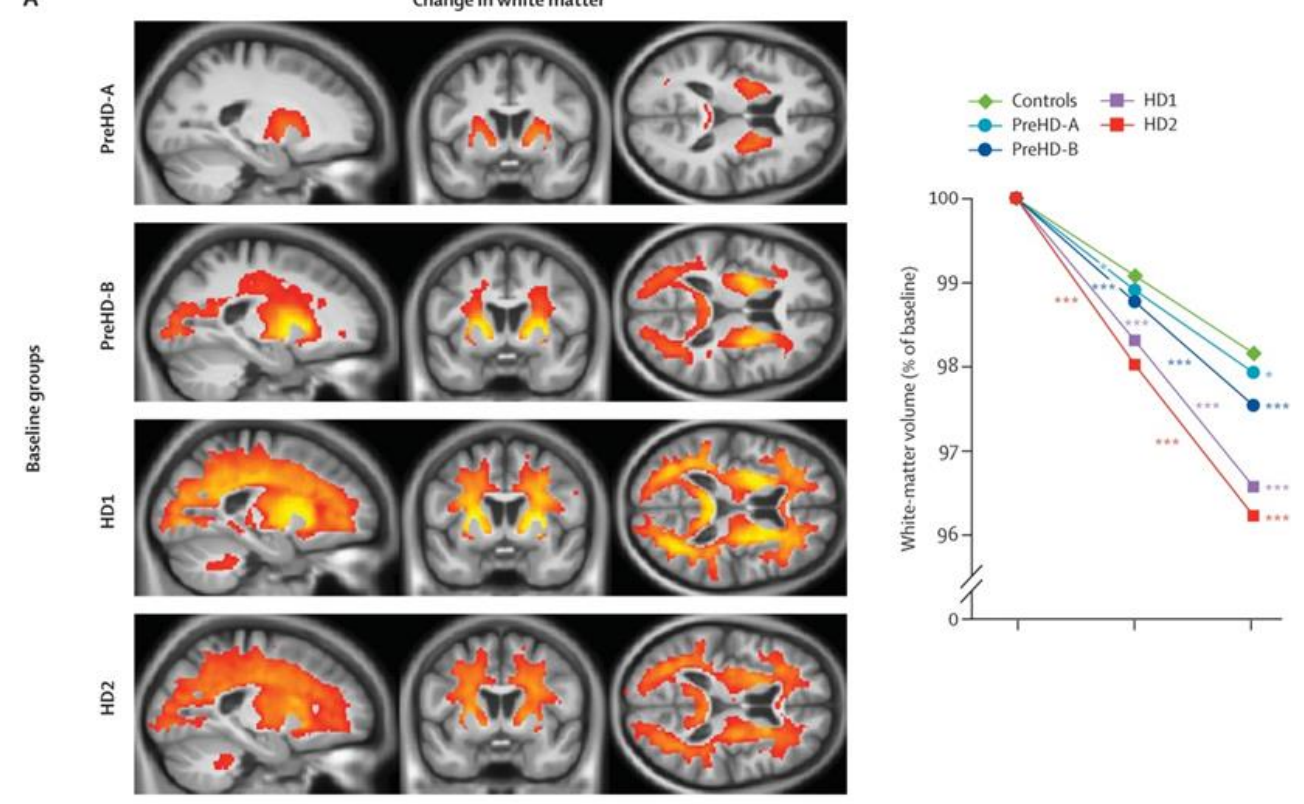

B
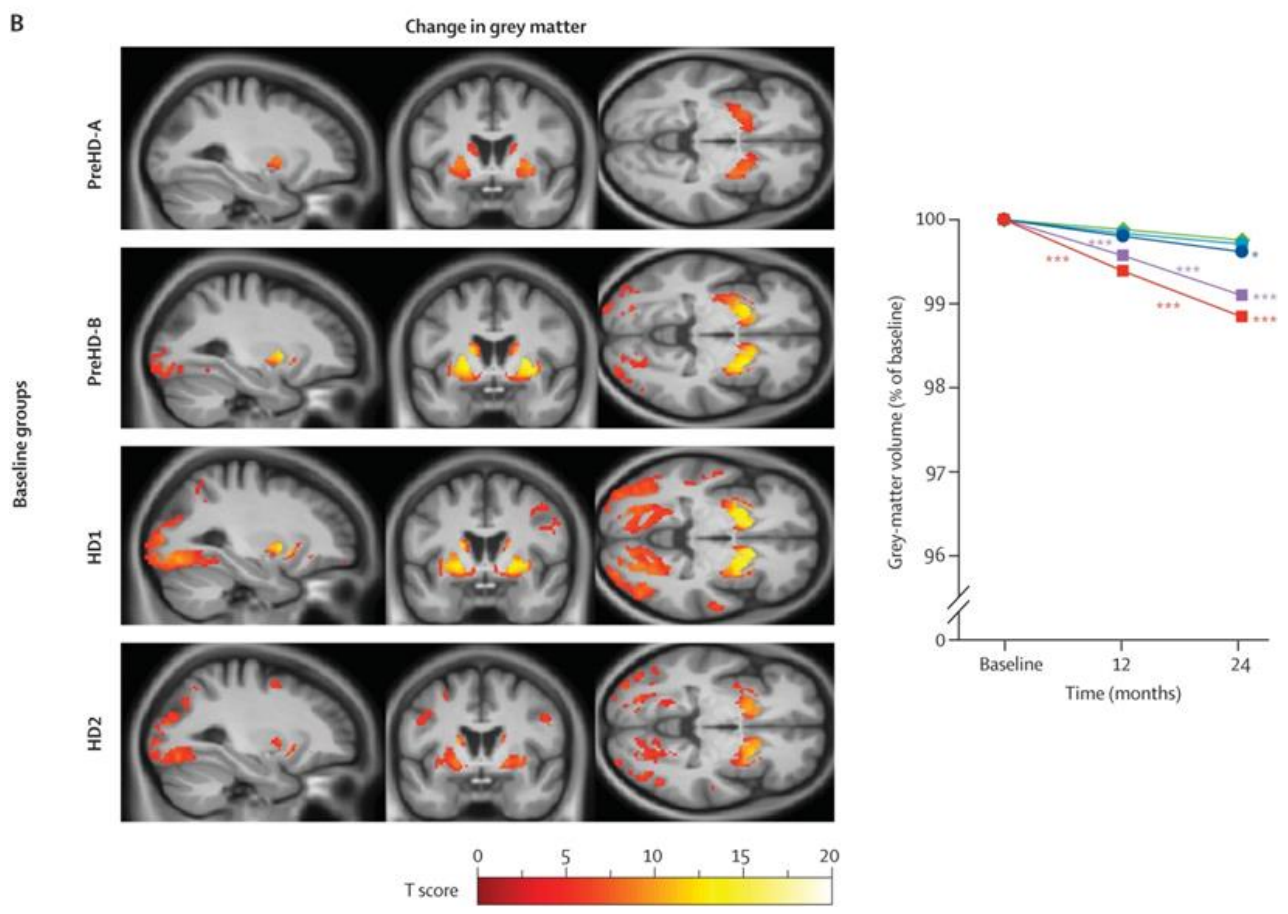

Figure 3: Longitudinal changes in grey and white matter. Parametric maps showing regions with statistically significant atrophy in (A) white matter and (B) grey matter in HD and pre-manifest brains over 24 months, relative to controls. Adjusted for age, sex, study site, and scan interval and corrected for multiple comparisons, $\mathrm{p}<0 \cdot 05$ ( FWE). Corresponding longitudinal plots show mean values at baseline, 12 months, and 24 months. Significant change differences relative to controls over $0-12,12-$ 24 , and $0-24$ months are represented by ${ }^{*} \mathrm{p}<0 \cdot 05,{ }^{* *} \mathrm{p}<0 \cdot 01$, and ${ }^{* * *} \mathrm{p}<0 \cdot 00$. preHD = premanifest HD, HD = early manifst HD. Reproduced from Lancet Neurology, Tabrizi et al, 2012 with permission from Elsevier 


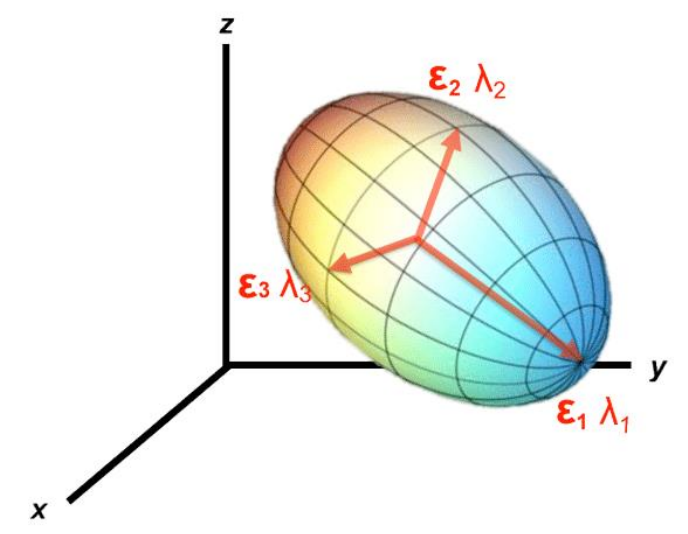

Figure 4: The Diffusion Tensor. The diffusion tensor is characterised by three eigenvalues $\left(\lambda_{1}, \lambda_{2}, \lambda_{3}\right)$ which represent diffusivity along the three principal axes and three eigenvectors $\left(\varepsilon_{1}, \varepsilon_{2}, \varepsilon_{3}\right)$ which represent corresponding orientation. The Trace is the sum of the eigenvalues $(\lambda 1+\lambda 2+\lambda 3)$; the average is mean diffusivity. Reproduced from mriquestions.com, with permission from Allen Elster, 2016.

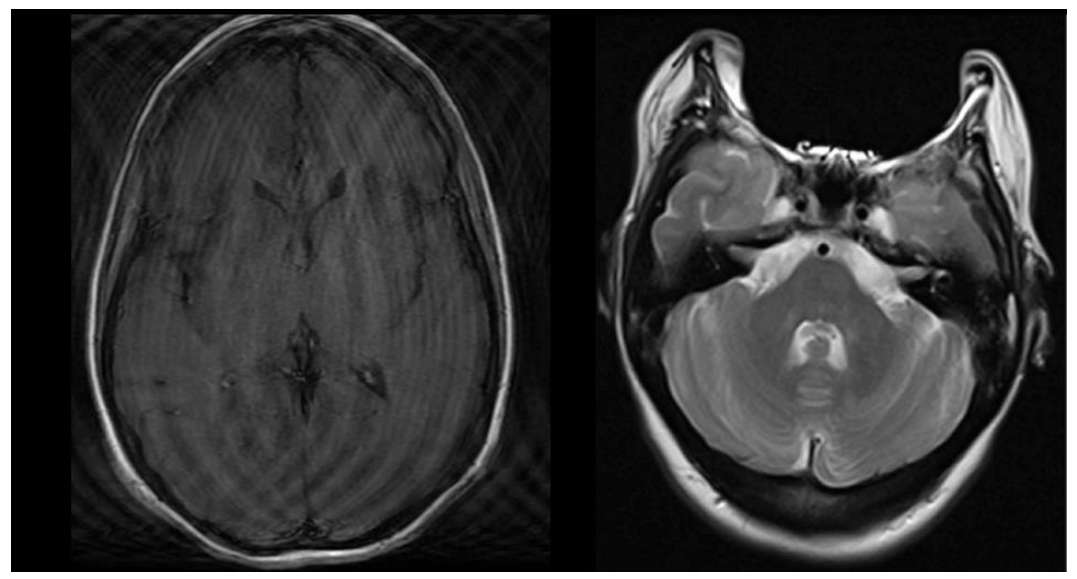

Figure 5: MRI Artefacts. a) Example of a motion artefact; b) Example of a susceptibility artefact caused by the presence of dental braces. Reproduced from svuhradiology.ie/diagnostic-imaging with permission from Eric Heffernan, 2016 
a)

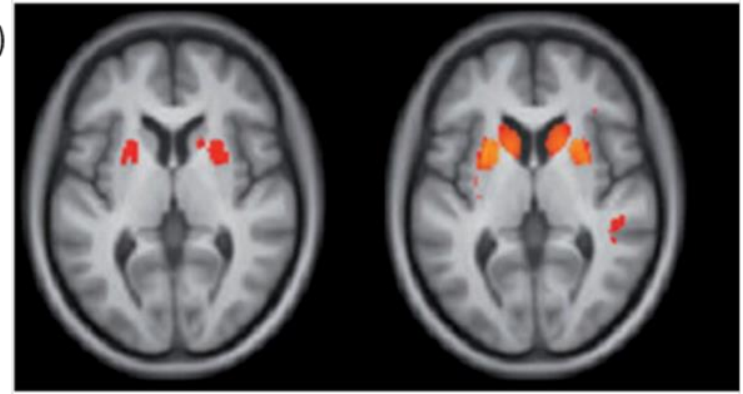

b) $\mathbf{A}$

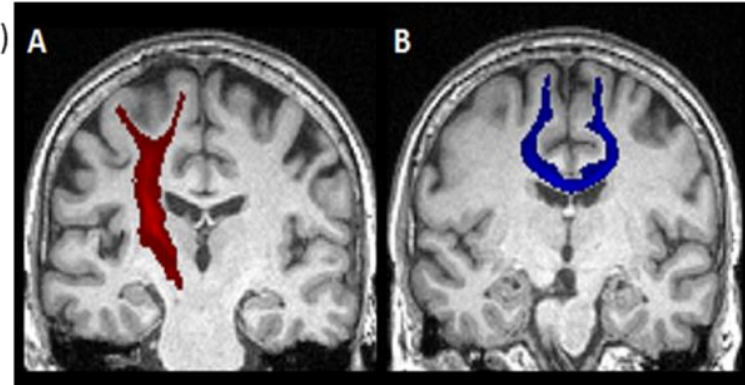

c)

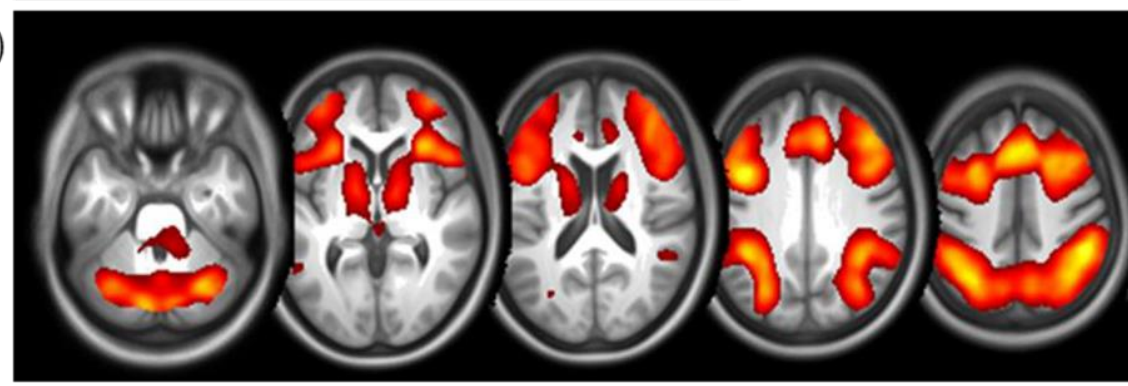

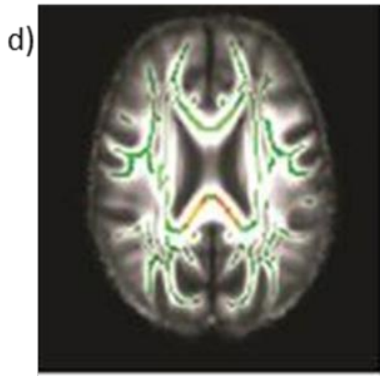

Figure 6: Examples of MRI data analysis. a)Voxel-based morphometry: statistical parametric maps of grey and white matter differences among groups compared with controls. Data adjusted for age, sex, study site, and intracranial volume. Corrected for multiple comparisons, $\mathrm{p}<0 \cdot 05$ (FWE). Reproduced from Lancet Neurology, Tabrizi et al, 2009 with permission from Elsevier; b) Tractography: exemplar reconstructed white matter tracts in the motor network; c) Task fMRI: increased working memory task activations (2-back vs. 0-back) in preHD, results shown at $\mathrm{p}<0.05$ after FWE-correction overlaid on normalized structural scan. Reproduced from EBioMedicine, Kloeppel et al, 2011,with permission from Open Access Elsevier. d) Tract Based Spatial Statistics: tracts in which reduced FA was correlated with increased levels of depression in those with high CPO; results corrected at $\mathrm{p}<0.05$ (FWE) (red-yellow) are projected on a white matter skeleton (green), overlaid on a customized mean FA image. Reproduced from the Journal of Huntington's disease, Gregory et al, 2015 with permission from IOS Press; d) Task fMRI: increased working memory task activations (2-back vs. 0-back) in preHD, results shown at $\mathrm{p}<0 \cdot 05$ after FWE-correction overlaid on normalized structural scan. Reproduced from EBioMedicine, Kloeppel et al, 2011,with permission from Open Access Elsevier. 


\section{References}

1. Ashburner J, Friston KJ (2000) Voxel-based morphometry--the methods. Neurolmage 11 (6 Pt

1):805-821. doi:10.1006/nimg.2000.0582

2. Ashburner J, Friston KJ (2001) Why voxel-based morphometry should be used. Neurolmage 14 (6):1238-1243. doi:10.1006/nimg.2001.0961

3. Morey RA, Petty CM, Xu Y, Hayes JP, Wagner HR, 2nd, Lewis DV, LaBar KS, Styner M, McCarthy G (2009) A comparison of automated segmentation and manual tracing for quantifying hippocampal and amygdala volumes. Neurolmage 45 (3):855-866. doi:10.1016/j.neuroimage.2008.12.033

4. Aylward EH, Codori AM, Rosenblatt A, Sherr M, Brandt J, Stine OC, Barta PE, Pearlson GD, Ross CA (2000) Rate of caudate atrophy in presymptomatic and symptomatic stages of Huntington's disease. Movement disorders : official journal of the Movement Disorder Society 15 (3):552-560

5. Aylward EH, Nopoulos PC, Ross CA, Langbehn DR, Pierson RK, Mills JA, Johnson HJ, Magnotta VA, Juhl AR, Paulsen JS (2011) Longitudinal change in regional brain volumes in prodromal Huntington disease. Journal of Neurology, Neurosurgery and Psychiatry 82 (4):405-410

6. Georgiou-Karistianis N, Scahill R, Tabrizi SJ, Squitieri F, Aylward E (2013) Structural MRI in Huntington's disease and recommendations for its potential use in clinical trials. Neuroscience and biobehavioral reviews 37 (3):480-490. doi:10.1016/j.neubiorev.2013.01.022

7. Paulsen JS, Nopoulos PC, Aylward E, Ross CA, Johnson H, Magnotta VA, Juhl A, Pierson RK, Mills J, Langbehn D, Nance M, Investigators P-H, Coordinators of the Huntington's Study G (2010) Striatal and white matter predictors of estimated diagnosis for Huntington disease. Brain research bulletin 82 (3-4):201-207. doi:10.1016/j.brainresbull.2010.04.003

8. Tabrizi SJ, Langbehn DR, Leavitt BR, Roos RA, Durr A, Craufurd D, Kennard C, Hicks SL, Fox NC, Scahill RI, Borowsky B, Tobin AJ, Rosas HD, Johnson H, Reilmann R, Landwehrmeyer B, Stout JC, investigators T-H (2009) Biological and clinical manifestations of Huntington's disease in the longitudinal TRACK-HD study: cross-sectional analysis of baseline data. Lancet neurology 8 (9):791801. doi:10.1016/S1474-4422(09)70170-X

9. Tabrizi SJ, Reilmann R, Roos RA, Durr A, Leavitt B, Owen G, Jones R, Johnson H, Craufurd D, Hicks SL, Kennard C, Landwehrmeyer B, Stout JC, Borowsky B, Scahill RI, Frost C, Langbehn DR, investigators T-H (2012) Potential endpoints for clinical trials in premanifest and early Huntington's 
disease in the TRACK-HD study: analysis of 24 month observational data. Lancet neurology 11 (1):4253. doi:10.1016/S1474-4422(11)70263-0

10. Tabrizi SJ, Scahill RI, Durr A, Roos RA, Leavitt BR, Jones R, Landwehrmeyer GB, Fox NC, Johnson H, Hicks SL, Kennard C, Craufurd D, Frost C, Langbehn DR, Reilmann R, Stout JC (2011) Biological and clinical changes in premanifest and early stage Huntington's disease in the TRACK-HD study: the 12month longitudinal analysis. Lancet neurology 10 (1):31-42

11. Tabrizi SJ, Scahill RI, Owen G, Durr A, Leavitt BR, Roos RA, Borowsky B, Landwehrmeyer B, Frost C, Johnson H, Craufurd D, Reilmann R, Stout JC, Langbehn DR (2013) Predictors of phenotypic progression and disease onset in premanifest and early-stage Huntington's disease in the TRACK-HD study: analysis of 36-month observational data. The Lancet Neurology

12. Rosas HD, Reuter M, Doros G, Lee SY, Triggs T, Malarick K, Fischl B, Salat DH, Hersch SM (2011) A tale of two factors: What determines the rate of progression in Huntington's disease? A longitudinal MRI study. Movement Disorders 26 (9):1691-1697

13. Johnson EB, Rees EM, Labuschagne I, Durr A, Leavitt BR, Roos RA, Reilmann R, Johnson H, Hobbs NZ, Langbehn DR, Stout JC, Tabrizi SJ, Scahill RI, investigators T-H (2015) The impact of occipital lobe cortical thickness on cognitive task performance: An investigation in Huntington's Disease.

Neuropsychologia 79 (Pt A):138-146. doi:10.1016/j.neuropsychologia.2015.10.033

14. Jones DK (2008) Studying connections in the living human brain with diffusion MRI. Cortex; a journal devoted to the study of the nervous system and behavior 44 (8):936-952 .

doi:10.1016/j.cortex.2008.05.002

15. Jones DK, Knosche TR, Turner R (2013) White matter integrity, fiber count, and other fallacies: the do's and don'ts of diffusion MRI. Neurolmage 73:239-254.

doi:10.1016/j.neuroimage.2012.06.081

16. Beaulieu C (2002) The basis of anisotropic water diffusion in the nervous system - A technical review. NMR Biomed 15 (7-8):435-455

17. Basser PJ, Mattiello J, LeBihan D (1994) MR diffusion tensor spectroscopy and imaging. Biophys J 66 (1):259-267. doi:10.1016/S0006-3495(94)80775-1

18. Smith SM, Jenkinson M, Johansen-Berg H, Rueckert D, Nichols TE, Mackay CE, Watkins KE, Ciccarelli O, Cader MZ, Matthews PM, Behrens TE (2006) Tract-based spatial statistics: voxelwise 
analysis of multi-subject diffusion data. Neurolmage 31 (4):1487-1505.

doi:10.1016/j.neuroimage.2006.02.024

19. Conturo TE, Lori NF, Cull TS, Akbudak E, Snyder AZ, Shimony JS, McKinstry RC, Burton H, Raichle ME (1999) Tracking neuronal fiber pathways in the living human brain. Proc Natl Acad Sci U S A 96 (18):10422-10427

20. Bullmore ET, Bassett DS (2011) Brain graphs: graphical models of the human brain connectome. Annu Rev Clin Psychol 7:113-140. doi:10.1146/annurev-clinpsy-040510-143934

21. Sporns O (2011) The human connectome: a complex network. Ann N Y Acad Sci 1224:109-125. doi:10.1111/j.1749-6632.2010.05888.x

22. Sporns O, Tononi G, Kotter R (2005) The human connectome: A structural description of the human brain. PLoS Comput Biol 1 (4):e42. doi:10.1371/journal.pcbi.0010042

23. Bohanna I, Georgiou-Karistianis N, Hannan AJ, Egan GF (2008) Magnetic resonance imaging as an approach towards identifying neuropathological biomarkers for Huntington's disease. Brain Research Reviews 58 (1):209-225

24. Della Nave R, Ginestroni A, Tessa C, Giannelli M, Piacentini S, Filippi M, Mascalchi M (2010) Regional distribution and clinical correlates of white matter structural damage in Huntington disease: a tract-based spatial statistics study. AJNR American journal of neuroradiology 31 (9):16751681. doi:10.3174/ajnr.A2128

25. Douaud G, Behrens TE, Poupon C, Cointepas Y, Jbabdi S, Gaura V, Golestani N, Krystkowiak P, Verny C, Damier P, Bachoud-Lévi AC, Hantraye P, Remy P (2009) In vivo evidence for the selective subcortical degeneration in Huntington's disease. Neurolmage 46 (4):958-966

26. Dumas EM, van den Bogaard SJ, Ruber ME, Reilman RR, Stout JC, Craufurd D, Hicks SL, Kennard C, Tabrizi SJ, van Buchem MA, van der Grond J, Roos RA (2012) Early changes in white matter pathways of the sensorimotor cortex in premanifest Huntington's disease. Human brain mapping 33 (1):203212. doi:10.1002/hbm.21205

27. Novak MJU, Seunarine KK, Gibbard CR, Hobbs NZ, Scahill RI, Clark CA, Tabrizi SJ (2014) White matter integrity in premanifest and early Huntington's disease is related to caudate loss and disease progression. Cortex; a journal devoted to the study of the nervous system and behavior 52 (1):98112 
28. Poudel GR, Stout JC, Dominguez DJ, Churchyard A, Chua P, Egan GF, Georgiou-Karistianis N (2015) Longitudinal change in white matter microstructure in Huntington's disease: The IMAGE-HD study. Neurobiology of disease 74:406-412. doi:10.1016/j.nbd.2014.12.009

29. Poudel GR, Stout JC, Dominguez DJ, Salmon L, Churchyard A, Chua P, Georgiou-Karistianis N, Egan GF (2014) White matter connectivity reflects clinical and cognitive status in Huntington's disease. Neurobiology of disease 65:180-187. doi:10.1016/j.nbd.2014.01.013

30. Gregory S, Cole JH, Farmer RE, Rees EM, Roos RA, Sprengelmeyer R, Durr A, Landwehrmeyer B, Zhang H, Scahill RI, Tabrizi SJ, Frost C, Hobbs NZ (2015) Longitudinal Diffusion Tensor Imaging Shows Progressive Changes in White Matter in Huntington's Disease. J Huntingtons Dis 4 (4):333-346. doi:10.3233/JHD-150173

31. Klöppel S, Draganski B, Golding CV, Chu C, Nagy Z, Cook PA, Hicks SL, Kennard C, Alexander DC, Parker GJM, Tabrizi SJ, Frackowiak RSJ (2008) White matter connections reflect changes in voluntaryguided saccades in pre-symptomatic Huntington's disease. Brain 131 (1):196-204. doi:10.1093/brain/awm275

32. McColgan P, Seunarine KK, Razi A, Cole JH, Gregory S, Durr A, Roos RA, Stout JC, Landwehrmeyer B, Scahill RI, Clark CA, Rees G, Tabrizi SJ, Track HDI (2015) Selective vulnerability of Rich Club brain regions is an organizational principle of structural connectivity loss in Huntington's disease. Brain 138 (Pt 11):3327-3344. doi:10.1093/brain/awv259

33. Orth M, Gregory S, Scahill RI, Mayer IS, Minkova L, Kloppel S, Seunarine KK, Boyd L, Borowsky B, Reilmann R, Bernhard Landwehrmeyer G, Leavitt BR, Roos RA, Durr A, Rees G, Rothwell JC, Langbehn D, Tabrizi SJ, Investigators TR-O (2016) Natural variation in sensory-motor white matter organization influences manifestations of Huntington's disease. Human brain mapping. doi:10.1002/hbm.23332

34. Odish OF, Caeyenberghs K, Hosseini H, van den Bogaard SJ, Roos RA, Leemans A (2015) Dynamics of the connectome in Huntington's disease: A longitudinal diffusion MRI study. Neurolmage Clinical 9:32-43. doi:10.1016/j.nicl.2015.07.003

35. Odish OF, Leemans A, Reijntjes RH, van den Bogaard SJ, Dumas EM, Wolterbeek R, Tax CM, Kuijf HJ, Vincken KL, van der Grond J, Roos RA (2015) Microstructural brain abnormalities in Huntington's disease: A two-year follow-up. Human brain mapping 36 (6):2061-2074. doi:10.1002/hbm.22756

36. Gregory S, Scahill RI, Seunarine KK, Stopford C, Zhang H, Zhang J, Orth M, Durr A, Roos RA, Langbehn DR, Long JD, Johnson H, Rees G, Tabrizi SJ, Craufurd D, Track HDI (2015) Neuropsychiatry 
and White Matter Microstructure in Huntington's Disease. J Huntingtons Dis 4 (3):239-249. doi:10.3233/JHD-150160

37. Ogawa S, Lee TM, Kay AR, Tank DW (1990) Brain magnetic resonance imaging with contrast dependent on blood oxygenation. Proc Natl Acad Sci U S A 87 (24):9868-9872

38. Ogawa S, Menon RS, Tank DW, Kim SG, Merkle H, Ellermann JM, Ugurbil K (1993) Functional brain mapping by blood oxygenation level-dependent contrast magnetic resonance imaging. $A$ comparison of signal characteristics with a biophysical model. Biophys J 64 (3):803-812. doi:10.1016/S0006-3495(93)81441-3

39. Bandettini PA, Wong EC, Hinks RS, Tikofsky RS, Hyde JS (1992) Time course EPI of human brain function during task activation. Magnetic resonance in medicine : official journal of the Society of Magnetic Resonance in Medicine / Society of Magnetic Resonance in Medicine 25 (2):390-397

40. Logothetis NK, Pfeuffer J (2004) On the nature of the BOLD fMRI contrast mechanism. Magn Reson Imaging 22 (10):1517-1531. doi:10.1016/j.mri.2004.10.018

41. Kim J, Zhu W, Chang L, Bentler PM, Ernst T (2007) Unified structural equation modeling approach for the analysis of multisubject, multivariate functional MRI data. Human brain mapping 28 (2):8593. doi:10.1002/hbm.20259

42. Seth $A K$, Barrett AB, Barnett $L$ (2015) Granger causality analysis in neuroscience and neuroimaging. J Neurosci 35 (8):3293-3297. doi:10.1523/JNEUROSCI.4399-14.2015

43. Friston KJ, Harrison L, Penny W (2003) Dynamic causal modelling. Neurolmage 19 (4):1273-1302 44. Georgiou-Karistianis N, Poudel GR, Dominguez DJ, Langmaid R, Gray MA, Churchyard A, Chua P, Borowsky B, Egan GF, Stout JC (2013) Functional and connectivity changes during working memory in Huntington's disease: 18 month longitudinal data from the IMAGE-HD study. Brain Cogn 83 (1):8091. doi:10.1016/j.bandc.2013.07.004

45. Poudel GR, Stout JC, Dominguez DJ, Gray MA, Salmon L, Churchyard A, Chua P, Borowsky B, Egan GF, Georgiou-Karistianis N (2015) Functional changes during working memory in Huntington's disease: 30-month longitudinal data from the IMAGE-HD study. Brain Struct Funct 220 (1):501-512. doi:10.1007/s00429-013-0670-z

46. Wolf RC, Kloppel S (2013) Clinical significance of frontal cortex abnormalities in Huntington's disease. Experimental neurology 247:39-44. doi:10.1016/j.expneurol.2013.03.022 
47. Wolf RC, Sambataro F, Vasic N, Depping MS, Thomann PA, Landwehrmeyer GB, Sussmuth SD, Orth M (2014) Abnormal resting-state connectivity of motor and cognitive networks in early manifest Huntington's disease. Psychological medicine 44 (15):3341-3356.

doi:10.1017/S0033291714000579

48. Wolf RC, Sambataro F, Vasic N, Schonfeldt-Lecuona C, Ecker D, Landwehrmeyer B (2008) Aberrant connectivity of lateral prefrontal networks in presymptomatic Huntington's disease. Experimental neurology 213 (1):137-144. doi:10.1016/j.expneurol.2008.05.017

49. Wolf RC, Sambataro F, Vasic N, Wolf ND, Thomann PA, Landwehrmeyer GB, Orth M (2014) Longitudinal task-negative network analyses in preclinical Huntington's disease. Eur Arch Psychiatry Clin Neurosci 264 (6):493-505. doi:10.1007/s00406-013-0447-7

50. Kloppel S, Draganski B, Siebner HR, Tabrizi SJ, Weiller C, Frackowiak RS (2009) Functional compensation of motor function in pre-symptomatic Huntington's disease. Brain 132 (Pt 6):16241632. doi:10.1093/brain/awp081

51. Gray MA, Egan GF, Ando A, Churchyard A, Chua P, Stout JC, Georgiou-Karistianis N (2013) Prefrontal activity in Huntington's disease reflects cognitive and neuropsychiatric disturbances: the IMAGE-HD study. Experimental neurology 239:218-228. doi:10.1016/j.expneurol.2012.10.020

52. Malejko K, Weydt P, Sussmuth SD, Gron G, Landwehrmeyer BG, Abler B (2014) Prodromal Huntington disease as a model for functional compensation of early neurodegeneration. PloS one 9 (12):e114569. doi:10.1371/journal.pone.0114569

53. Kloppel S, Gregory, S. (2015) Compensation in Preclinical Huntington's Disease: Evidence From the Track-On HD Study. EBioMedicine. doi:http://dx.doi.org/10.1016/i.ebiom.2015.08.002 54. Harrington DL, Rubinov M, Durgerian S, Mourany L, Reece C, Koenig K, Bullmore E, Long JD, Paulsen JS, Group P-HiotHS, Rao SM (2015) Network topology and functional connectivity disturbances precede the onset of Huntington's disease. Brain 138 (Pt 8):2332-2346. doi:10.1093/brain/awv145

55. Werner CJ, Dogan I, Sass C, Mirzazade S, Schiefer J, Shah NJ, Schulz JB, Reetz K (2014) Altered resting-state connectivity in Huntington's disease. Human brain mapping 35 (6):2582-2593. doi:10.1002/hbm.22351

56. Odish OF, van den Berg-Huysmans AA, van den Bogaard SJ, Dumas EM, Hart EP, Rombouts SA, van der Grond J, Roos RA, Group T-HI (2015) Longitudinal resting state fMRI analysis in healthy 
controls and premanifest Huntington's disease gene carriers: a three-year follow-up study. Human brain mapping 36 (1):110-119. doi:10.1002/hbm.22616

57. Mumford JA (2012) A power calculation guide for fMRI studies. Soc Cogn Affect Neurosci 7 (6):738-742. doi:10.1093/scan/nss059

58. Button KS, loannidis JP, Mokrysz C, Nosek BA, Flint J, Robinson ES, Munafo MR (2013) Power failure: why small sample size undermines the reliability of neuroscience. Nat Rev Neurosci 14 (5):365-376. doi:10.1038/nrn3475

59. Pernet C, Poline JB (2015) Improving functional magnetic resonance imaging reproducibility. GigaScience 4:15. doi:10.1186/s13742-015-0055-8

60. Mansfield P (1984) Real-time echo-planar imaging by NMR. Br Med Bull 40 (2):187-190

61. Sled JG, Zijdenbos AP, Evans AC (1998) A nonparametric method for automatic correction of intensity nonuniformity in MRI data. leee Transactions on Medical Imaging 17 (1):87-97. doi:Doi $10.1109 / 42.668698$

62. Ashburner J, Friston KJ (2005) Unified segmentation. Neurolmage 26 (3):839-851. doi:10.1016/j.neuroimage.2005.02.018

63. Fischl B, Dale AM (2000) Measuring the thickness of the human cerebral cortex from magnetic resonance images. Proc Natl Acad Sci U S A 97 (20):11050-11055. doi:10.1073/pnas.200033797

64. Fischl B, Salat DH, Busa E, Albert M, Dieterich M, Haselgrove C, van der Kouwe A, Killiany R, Kennedy D, Klaveness S, Montillo A, Makris N, Rosen B, Dale AM (2002) Whole brain segmentation: automated labeling of neuroanatomical structures in the human brain. Neuron 33 (3):341-355

65. Fischl B, van der Kouwe A, Destrieux C, Halgren E, Segonne F, Salat DH, Busa E, Seidman LJ, Goldstein J, Kennedy D, Caviness V, Makris N, Rosen B, Dale AM (2004) Automatically parcellating the human cerebral cortex. Cerebral cortex 14 (1):11-22

66. Andersson JL, Sotiropoulos SN (2016) An integrated approach to correction for off-resonance effects and subject movement in diffusion MR imaging. Neurolmage 125:1063-1078. doi:10.1016/j.neuroimage.2015.10.019

67. Lori NF, Akbudak E, Shimony JS, Cull TS, Snyder AZ, Guillory RK, Conturo TE (2002) Diffusion tensor fiber tracking of human brain connectivity: aquisition methods, reliability analysis and biological results. NMR Biomed 15 (7-8):494-515. doi:10.1002/nbm.779 
68. Friston KJ, Worsley KJ, Frackowiak RS, Mazziotta JC, Evans AC (1994) Assessing the significance of focal activations using their spatial extent. Human brain mapping 1 (3):210-220.

doi:10.1002/hbm.460010306

69. Worsley KJ, Marrett S, Neelin P, Vandal AC, Friston KJ, Evans AC (1996) A unified statistical approach for determining significant signals in images of cerebral activation. Human brain mapping 4 (1):58-73. doi:10.1002/(SICI)1097-0193(1996)4:1\&lt;58::AID-HBM4\&gt;3.0.CO;2-O

10.1002/(SICI)1097-0193(1996)4:1<58::AID-HBM4>3.0.CO;2-O

70. Smith SM, Nichols TE (2009) Threshold-free cluster enhancement: addressing problems of smoothing, threshold dependence and localisation in cluster inference. Neurolmage 44 (1):83-98. doi:10.1016/j.neuroimage.2008.03.061

71. Khalsa S, Mayhew SD, Chechlacz M, Bagary M, Bagshaw AP (2014) The structural and functional connectivity of the posterior cingulate cortex: comparison between deterministic and probabilistic tractography for the investigation of structure-function relationships. Neurolmage $102 \mathrm{Pt}$ 1:118-127. doi:10.1016/j.neuroimage.2013.12.022

72. Biswal B, Yetkin FZ, Haughton VM, Hyde JS (1995) Functional connectivity in the motor cortex of resting human brain using echo-planar MRI. Magnetic resonance in medicine : official journal of the Society of Magnetic Resonance in Medicine / Society of Magnetic Resonance in Medicine 34 (4):537541

73. Fox MD, Snyder AZ, Vincent JL, Corbetta M, Van Essen DC, Raichle ME (2005) The human brain is intrinsically organized into dynamic, anticorrelated functional networks. Proc Natl Acad Sci U S A 102 (27):9673-9678. doi:10.1073/pnas.0504136102

74. Gotts SJ, Saad ZS, Jo HJ, Wallace GL, Cox RW, Martin A (2013) The perils of global signal regression for group comparisons: a case study of Autism Spectrum Disorders. Front Hum Neurosci 7:356. doi:10.3389/fnhum.2013.00356

75. Jenkinson M, Smith S (2001) A global optimisation method for robust affine registration of brain images. Medical image analysis 5 (2):143-156

76. Qing Z, Dong Z, Li S, Zang Y, Liu D (2015) Global signal regression has complex effects on regional homogeneity of resting state fMRI signal. Magn Reson Imaging 33 (10):1306-1313.

doi:10.1016/j.mri.2015.07.011 
77. Calhoun VD, Adali T, Pearlson GD, Pekar JJ (2001) A method for making group inferences from functional MRI data using independent component analysis. Human brain mapping 14 (3):140-151 78. Beckmann CF, Smith SM (2004) Probabilistic independent component analysis for functional magnetic resonance imaging. IEEE Trans Med Imaging 23 (2):137-152. doi:10.1109/TMI.2003.822821 79. Filippini N, MacIntosh BJ, Hough MG, Goodwin GM, Frisoni GB, Smith SM, Matthews PM, Beckmann CF, Mackay CE (2009) Distinct patterns of brain activity in young carriers of the APOEepsilon4 allele. Proc Natl Acad Sci U S A 106 (17):7209-7214. doi:10.1073/pnas.0811879106 